Check for updates

Cite this: RSC Adv., 2017, 7, 56271

\title{
Long non-coding RNA expression profiles predict clinical phenotypes of seminoma and yolk sac tumort
}

\author{
Li Li, (DD *a Luo Qiang, ${ }^{a}$ Liu Yufeng, ${ }^{a}$ Zhang Lin ${ }^{\text {bcd }}$ and Yao Wu ${ }^{b}$
}

Malignant germ cell tumors (GCTs) such as seminoma and yolk sac tumor cause serious health problems but with favorable prognosis if they were diagnosed timely. To investigate potential biomarkers used for GCTs diagnosis and phenotype distinguishment, we first applied a IncRNA classification pipeline to identify 368 IncRNAs represented on the Affymetrix Human Genome U133A Array. We then comprehensively analyzed the IncRNA expression patterns in a set of previously published gene expression profiles of seminoma and yolk sac tumor stratified by different age groups (children and adults). The IncRNAs expression signatures between children and adults in different GCTs phenotypic groups were identified respectively, five aberrantly expressed IncRNAs were shared by children and adults, indicating a role for them in distinguishing seminoma from yolk sac tumor regardless of age. In parallel, nine distinctive IncRNAs were also determined between seminoma and yolk sac tumor, which suggested that people may face a high risk of suffering from GCTs. Our findings may contribute to the early diagnosis and prognosis of GCTs regardless of patients' age and other diseases.

Received 4th November 2017 Accepted 4th December 2017

DOI: 10.1039/c7ra12131h

rsc.li/rsc-advances and the malignant GCTs accounted for $2.9 \%$ of all malignant tumors in children who were younger than 15 years old worldwide. ${ }^{5}$ In general, GCTs are characterized by a high heterogeneity of their histological differentiation, but they show a similar histological pattern independent of their primary site or sex. ${ }^{6}$ As indicated by Teilum in 1965, the neoplastic cell that derived from gonadal or extragonadal germ cell was able to trans-differentiate into embryonal and exo-embryonal malignant carcinoma. ${ }^{7}$ The former includes mature/immature teratoma in embryo and choriocarcinoma ( $\mathrm{CHC}$ ) and yolk sac tumor (yolk sac tumor) outside the embryo. Meanwhile, the exoembryonal carcinoma such as seminoma (testis), dysgerminoma (ovary) and germinoma (brain) are all malignant tumors (Fig. 1).

In recent years, biomarkers including al-fetoprotein (AFP) and human choriogonadotropin (HCG) have been used for diagnosis of yolk sac tumor and $\mathrm{CHC}$, and a moderate elevation of $\beta$-HCG was considered to occur in seminoma. Despite great progress achieved in the early diagnosis and distinguishment of different clinical phenotypes of GCTs, a great amount of misdiagnosis still occurred every year. For example, the reference value of HCG used to diagnosis seminoma/germinoma $\left(<50\right.$ IU L $\left.{ }^{-1}\right)$ was similar to syncytiotrophoblast-like giant cells. ${ }^{8}$ Additionally, in neonates and young infants, the AFP was born with a physiologically elevated level, but children older than two years old with a high AFP level $\left(\geq 100 \mu \mathrm{g} \mathrm{L}^{-1}\right)$ can be considered as malignant GCTs. ${ }^{9}$ Nevertheless, in some liver diseases such as acute liver failure, hepatocellular carcinoma, and hepatoblastoma, the APF secretion is also elevated due to 


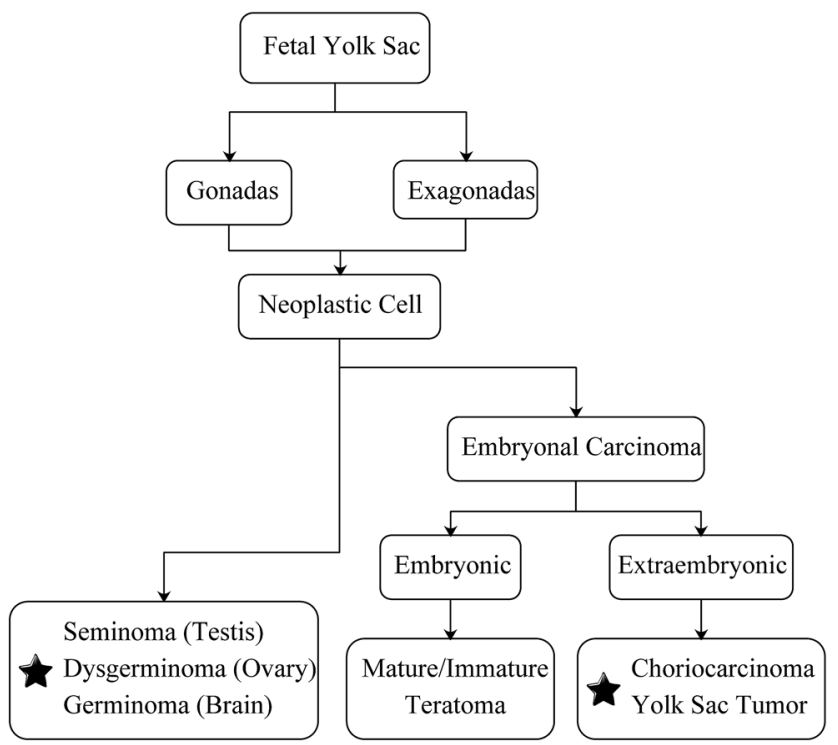

Fig. 1 Classification and development of germ cell tumors. Malignant tumors are labeled with pentagram.

hepatocellular regeneration..$^{10}$ Thus, it may lead to incorrect judgment to make clinical decisions only depending on images or molecular biomarkers, and it would be of great significance to find out more stable and accurate biomarkers that were used to diagnosis and distinguish different clinical phenotypes of GCTs regardless of patient age and the disturbance from other diseases.

The emerging role of long non-coding RNA (lncRNA) as promising biomarker and critical therapeutic target has drawn considerable attentions. However, the role of lncRNA in GCTs has not been investigated. Typically, IncRNAs are non-protein coding transcripts longer than 200 nucleotides which were involved in numerous critical biological processes such as $\mathrm{X}$ chromosome silencing, genomic imprinting, chromosome modification, transcriptional activation, transcriptional interference, and nuclear transport. ${ }^{11}$ HOTAIR, for example, a wellstudied IncRNA, was found aberrantly expressed in different subtypes of breast cancer, which highlighted the role of lncRNA in distinguishing breast cancer from different subtypes for the first time..$^{12}$ In glioma and colorectal cancer, lncRNAs such as HOXA-AS, MALAT1, and NEAT1 were all found to be specifically distributed. ${ }^{13,14}$ Hence it may be a new way to distinguish malignant GCTs from embryonal to exo-embryonal via lncRNA profiling. Favorably, microarray datasets shared by previous studies can be achieved from the Gene Expression Omnibus (GEO) and used to investigate our hypothesis.

Herein, we aimed at profiling lncRNA expression signatures in embryonal malignant carcinoma (yolk sac tumor) and exoembryonal malignant carcinoma (seminoma) by analyzing a cohort of previously published microarray datasets that achieved from the GEO. The distinctive IncRNAs were identified through comparison between groups of different age and GCTs phenotypes respectively. Our findings provide novel information on IncRNA expression profiles that may help to distinguish
GCTs from different phenotypes regardless of the limitation of age and disturbance from other diseases, and the results also provided potential diagnostic biomarkers and therapeutic targets for yolk sac tumor and seminoma.

\section{Materials and methods}

\section{GEO seminoma and yolk sac tumor expression data}

All experiments were performed in compliance with the guidelines approved by the ethics committee at the Memorial SloanKettering Cancer Center (New York, NY) between 1987 and 1999. Informed consents were obtained from human participants of this study. The microarray datasets of seminoma and yolk sac tumor related to children and adult were obtained from the GEO. To compare the IncRNA expression signatures according to patient of different age and GCT phenotypes, two panels of adult and pediatric GCT gene expression datasets were included in this study: GSE3218 and GSE10615. The raw files of these two datasets which were based on the platform of Affymetrix Human Genome U133A Array were downloaded from the GEO, the data quality control process including quartile normalization, background adjustment, and summarization was processed using the Robust Multichip Average software (RMA, 1.2.0 In Development), which has been proved to be more efficient in estimating IncRNA expression fold changes than other software. Also, samples with a median expression value that exceeded the control limit line in plots of normalized unscaled standard error (NUSE) and relative log expression (RLE) were excluded from the downstream analysis. With this, a set of probe ID-centric gene expression values was obtained.

\section{IncRNA classification pipeline}

To evaluate lncRNA expressions in the microarray datasets that were obtained from the above step, we adopted the IncRNA classification pipeline which had been previously described to identify lncRNAs represented on the Affymetrix Genome array. ${ }^{15}$ In brief, we first mapped the ID-centric gene expression matrix to the NetAffx Annotation File (HG-U133A Annotations, CSV format, Release 35, $7 \mathrm{MB}, 10 / 7 / 2014$ ), which was available on the Affymetrix official website (http://www.affymetrix.com). Next, we only retained probes that labeled as "NR_" in the column of RefSeq transcripts IDs. While in the Ensembl gene IDs column, we selected probes that labeled as "lincRNA," "processed_transcript," "macro_IncRNA" or "misc_RNA." Lastly, we filtered the extracted annotated IncRNAs to exclude pseudogenes, rRNAs, microRNAs or other short RNAs (tRNAs, snRNAs, and snoRNAs).

\section{Differentially expressed IncRNAs screening}

Gene-e software was used to determine the differentially expressed IncRNAs between seminoma and yolk sac tumor in adult and children. Similarly, the distinctive IncRNAs between adult and children stratified by GCTs phenotypes (seminoma or yolk sac tumor) were also investigated. Conditions used to screen the differentially expressed lncRNAs were set as follows: false discovery rate $(\mathrm{FDR})<20 \%$, fold change $\geq 2$, permutation 
time 1000 and $p$-value $<0.01$. The co-existed differentially expressed lncRNAs between seminoma and yolk sac tumor or adult and children were intersected using Venn diagram. In order to investigate the effectiveness of the pack mode, the principal component analysis (PCA) was adopted using $\mathrm{MeV}$ (available at http://mev.tm4.org).

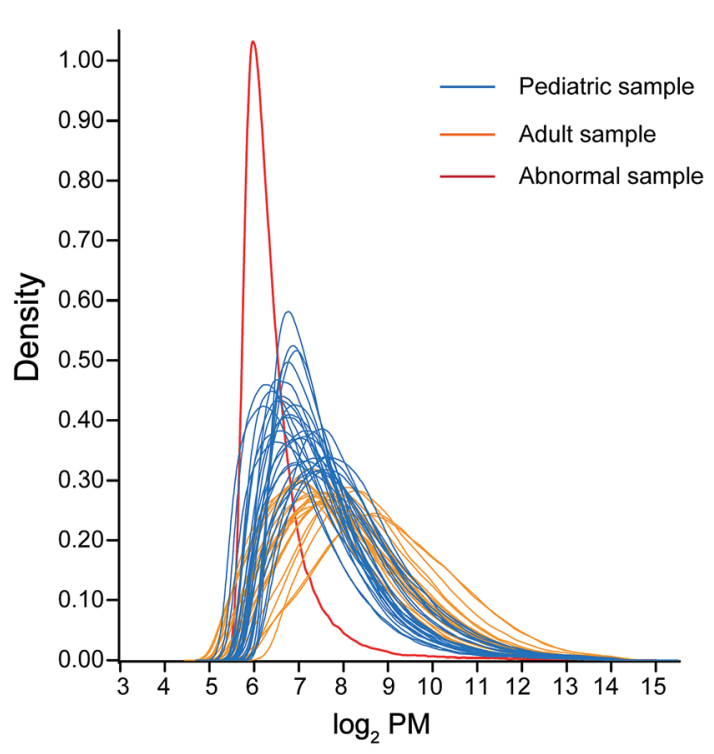

Fig. 2 Density plots of $\log _{2}$ PM by array. Pediatric samples were draw in blue, adult samples were shown in yellow, the abnormal sample which was excluded from the downstream analysis was shown in red.

\section{Validation of differentially expressed lncRNAs}

The Oncomine database which was hosted by Thermo Fisher Scientific Inc. provided more than 715 datasets and 86733 samples with expertly curated data. Thus we took advantage of this database to validate the expression of lncRNAs shared by adults and children, the comparison mode was selected as cancer $v s$. normal after uploading lncRNAs, and values of fold change, $t$ test, and $p$ statistics were recorded and used for further analysis.

\section{Statistical analysis}

All statistical analyses were processed using SAS version 9.2 for windows (SAS Institute Inc., Cary, North Carolina, USA). Differentially expressed lncRNAs were investigated using Genee software, PCA analysis was adopted using MeV online version. The age of children and adult with normal distribution were shown as mean \pm standard deviation (SD). A $p$ value less than 0.05 was considered as statistically significant unless otherwise specified.

\section{Results}

\section{Datasets characteristics}

The gene expression data of pediatric and adult seminoma and yolk sac tumors were included in this study: GES10615 and GSE3218A. The gene chip GSE10615 contained 28 pediatric samples, among which 18 samples were malignant yolk sac tumors, while 10 samples were malignant seminomas, including 1 seminoma sample which was excluded after quality

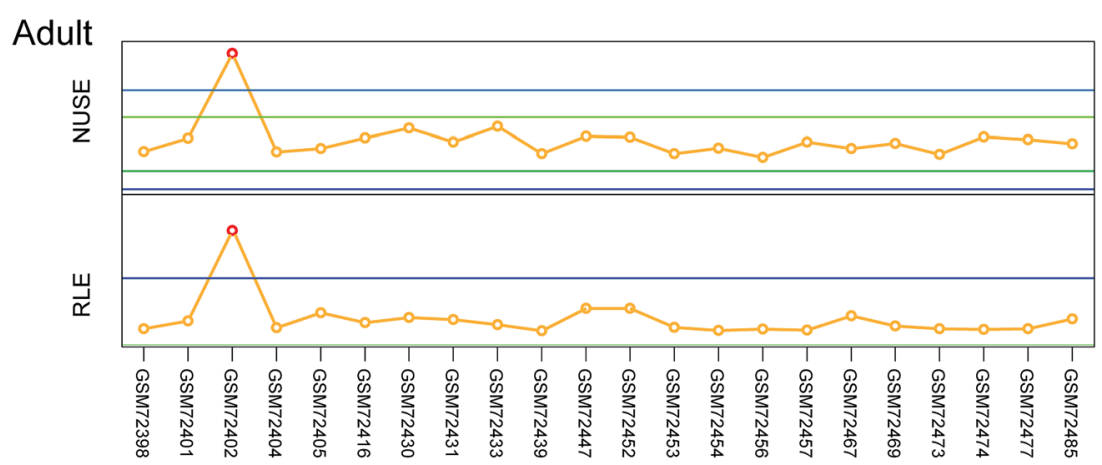

Children

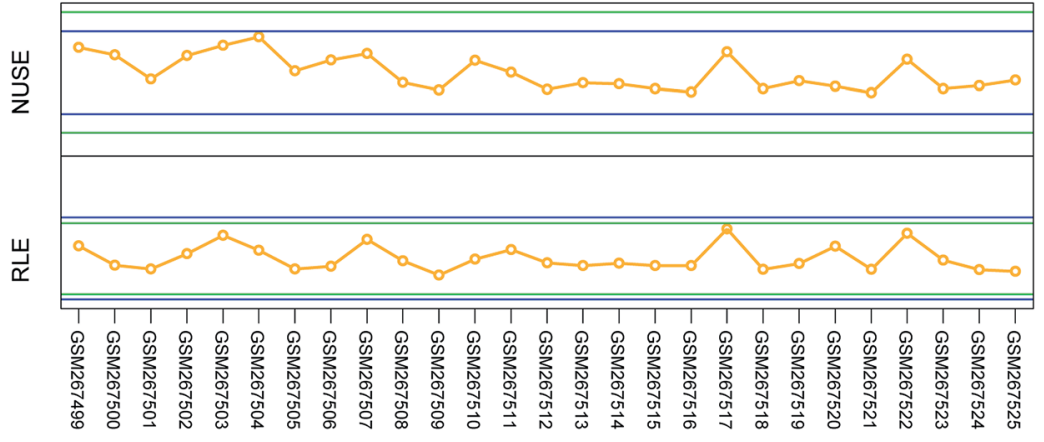

Fig. 3 NUSE and RLE median plots by array. The median of the normalized unscaled standard error (NUSE) and relative log expression (RLE) of every sample were evaluated in the expression quality control process, samples that exceeded the control limits (blue) were considered as low quality and would be excluded from the downstream analysis. 
Table 1 Differentially expressed IncRNAs between seminoma and yolk sac tumor

\begin{tabular}{|c|c|c|c|c|c|}
\hline Probesets & $\begin{array}{l}\text { RefSeq transcript } \\
\text { ID }\end{array}$ & Ensembl gene ID & Gene symbol & Regulation $^{a}$ & Gene title \\
\hline \multicolumn{6}{|l|}{ Adult } \\
\hline 221728_x_at & NR_001564 & ENSG00000274655 & XIST & Down & $\mathrm{X}$ (inactive)-specific transcript \\
\hline 214218_s_at & NR_001564 & ENSG00000274655 & XIST & Down & $\mathrm{X}$ (inactive)-specific transcript \\
\hline 207698_at & NR_026773 & ENSG00000146521 & C6orf123 & Down & Chromosome 6 open reading frame 123 \\
\hline 221621_at & NR_027058 & ENSG00000234912 & C17orf86 & Down & Chromosome 17 open reading frame 86 \\
\hline 220904_at & NR_026780 & ENSG00000231690 & C6orf208 & Up & Chromosome 6 open reading frame 208 \\
\hline 222001_x_at & NR_024510 & ENSG00000226067 & LOC728855 & Down & Hypothetical LOC728855 \\
\hline 220505_at & NR_024274 & ENSG00000224854 & C9orf53 & Down & Chromosome 9 open reading frame 53 \\
\hline 220904_at & NR_026780 & ENSG00000281305 & C6orf208 & Down & Chromosome 6 open reading frame 208 \\
\hline 214218_s_at & NR_001564 & ENSG00000229807 & XIST & Down & $\mathrm{X}$ (inactive)-specific transcript \\
\hline 215283_at & NR_015389 & ENSG00000263753 & LOC339290 & Up & Hypothetical LOC339290 \\
\hline 217506_at & NR_015389 & ENSG00000263753 & LOC339290 & Up & Hypothetical LOC339290 \\
\hline 219817_at & NR_015404 & ENSG00000234608 & C12orf47 & Down & Chromosome 12 open reading frame 47 \\
\hline \multicolumn{6}{|l|}{ Child } \\
\hline 221728_x_at & NR_001564 & ENSG00000274655 & XIST & Down & $\mathrm{X}$ (inactive)-specific transcript \\
\hline 214218_s_at & NR_001564 & ENSG00000274655 & XIST & Down & $\mathrm{X}$ (inactive)-specific transcript \\
\hline 206478_at & NR_026800 & ENSG00000226777 & KIAA0125 & Down & KIAA0125 \\
\hline 221621_at & NR_027058 & ENSG00000234912 & C17orf86 & Down & Chromosome 17 open reading frame 86 \\
\hline 206819_at & NR_003714 & ENSG00000197210 & POM121L9P & Down & POM121 membrane glycoprotein-like 9 \\
\hline 209917_s_at & NR_015381 & ENSG00000182165 & TP53TG1 & Down & TP53 target 1 \\
\hline 206819_at & NR_003714 & ENSG00000161103 & POM121L9P & Down & POM121 membrane glycoprotein-like 9 \\
\hline 220364_at & NR_027706 & ENSG00000278921 & FLJ11235 & Down & Hypothetical FLJ11235 \\
\hline 219817_at & NR_015404 & ENSG00000234608 & C12orf47 & Down & Chromosome 12 open reading frame 47 \\
\hline 220399_at & NR_024321 & ENSG00000272812 & NCRNA00115 & Down & Non-protein coding RNA 115 \\
\hline 219442_at & NR_024034 & ENSG00000276867 & C16orf67 & Up & Chromosome 16 open reading frame 67 \\
\hline 219442_at & NR_024034 & ENSG00000131797 & C16orf67 & Up & Chromosome 16 open reading frame 67 \\
\hline 214983_at & NR_001545 & ENSG00000233864 & TTTY15 & Up & Testis-specific transcript, Y-linked 15 \\
\hline
\end{tabular}

control (Fig. 2 and 3). In parallel, a total number of 21 adult samples including 9 yolk sac tumors and 12 seminomas were included in GES3218A. Each sample involved in this study was purely one phenotype of tumor specified, not mixed with others. The age of the children represented by GSE10615 was $8.3 \pm 5.6$ years old, while the related indicator was not obtained in the adult group.

\section{IncRNA expression profiles on Affymetrix Human Genome U133A Array}

With the lncRNA classification pipeline, 398 probe sets corresponding to 368 lncRNA genes were identified. Of these, 49 probe sets ( 40 genes) were annotated as lncRNAs by both RefSeq and Ensembl database, 267 probe sets (216 genes) were annotated by RefSeq database, and 180 probe sets (192 genes) were annotated by Ensembl database. In addition, probe sets that were annotated by both databases but had controversial definitions were excluded from this study (Tables S1 and S2†).

\section{Distinctive lncRNA expressions between seminoma and yolk sac tumor}

We compared the IncRNA expression patterns between seminoma and yolk sac tumor stratified by age. A total number of 13 probe sets corresponding to 11 lncRNA genes were identified in adults. Meanwhile, 13 probe sets corresponding to 12 genes were found aberrantly expressed in children, and 6 probe sets ( 5 
A

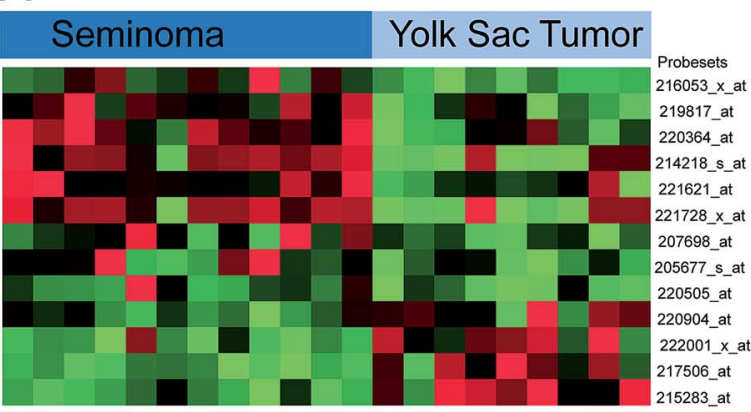

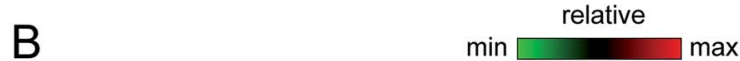

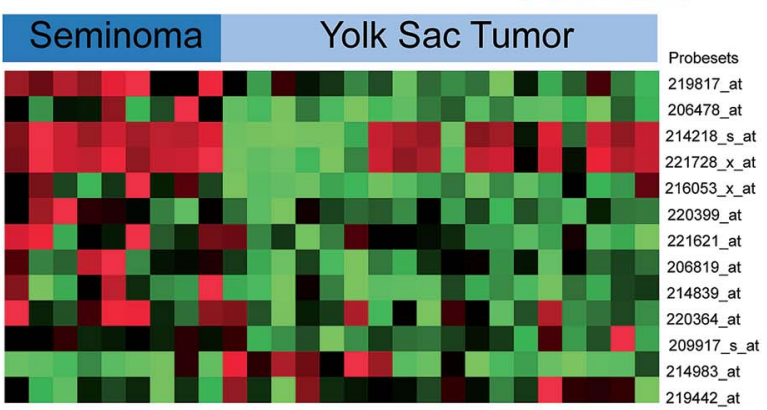

C

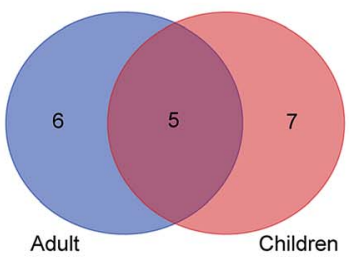

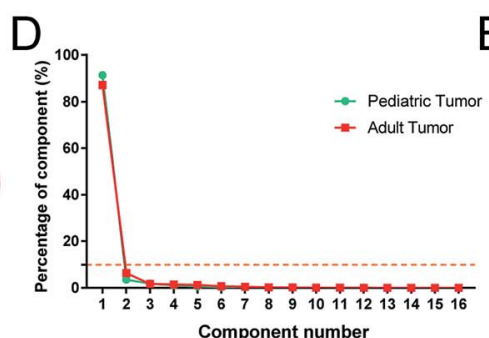

E

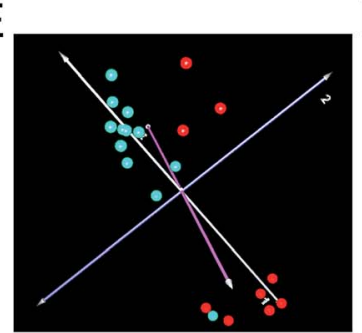

$\mathrm{F}$

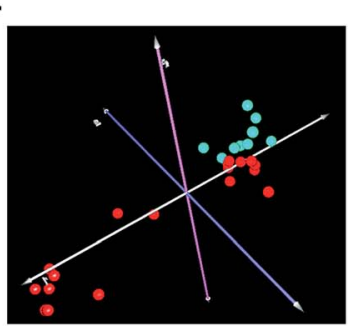

Fig. 4 Distinctive IncRNAs between seminoma and yolk sac tumor. Differentially expressed IncRNAs between seminoma and yolk sac tumor stratified by different age groups were investigated (adult: (A), children: (B)), the IncRNAs that were relatively high expressed were shown in red, the lower expressed ones were shown in green. (C) was a Venn diagram representing for the IncRNAs shared by adult and children. The results of principal component analysis were shown in (D-F). The percentage of every component was shown in (D), the lower control limit (10\%) for the principal component analysis were dotted in yellow, and two classes of samples corresponding to the dots of different colors in the 3D images were clustered ((E) adult, (F) children, seminoma: cyan, yolk sac tumor: red).

genes) including XIST, C17orf86, FAM182A, FLJ11235, and C12orf47 were shared by children and adult. Moreover, 19 probe sets corresponding to 16 lncRNA genes were identified between seminoma and yolk sac tumor when the effect of age was excluded (Table 1). Interestingly, five lncRNAs shared by adult and children were all involved in this cluster, and there were 10 differentially expressed lncRNAs that were overlapped in adult and pediatric lncRNA clusters. The PCA results of seminoma and yolk sac tumor were shown in Fig. 4, the cumulative component percentage reached up to $90 \%$ when the component number was set as 3 . However, it was much higher (more than $90 \%$ ) when the component number was set as 2 regardless of age, and dots with different colors of the same disease tightly clustered together.

\section{Distinctive IncRNA expressions between children and adult}

We also compared the IncRNA expression patterns between children and adult stratified by seminoma and yolk sac tumor. In seminoma, 16 probe sets corresponding to 14 lncRNAs were identified, while in yolk sac tumor, 17 probe sets corresponding to 16 lncRNAs were shown to be aberrantly expressed (Table 2). A total number of 9 lncRNAs such as PART1, NCRNA00230A, POM121L9P, MEG3, TP53TG1, LOC157627, LOC339290, DKFZP434L187, and TTTY15 were shared by seminoma and yolk sac tumor (Table 3). The PCA results of children and adult were shown in Fig. 5 with a validity $\geq 90 \%$, and 2 components were clustered, dots of the same age group were clustered together.

\section{Validation of differentially expressed lncRNAs}

The expression levels of lncRNAs shared by yolk sac tumor and seminoma were investigated using Oncomine database and compared between yolk sac tumor and seminoma, yolk sac tumor or seminoma and normal respectively. Apart from lncRNA FAM182A and FLJ11235 which cannot be found in Oncomine database, the expressions of lncRNA XIST and C17orf86 were all of statistical significance between cancer and normal except for C17orf86 in seminoma (Table 4), which were highly consistent with our assumptions.

\section{Discussion}

To date, different phenotypes of malignant GCTs were determined mainly depending on histological changes, but it may be misdiagnosed when facing patients of different ages or suffering from other diseases. As epidemiological and clinical evidence indicated, the histological changes of malignant GCTs among different clinical phenotypes were partly overlapped, ${ }^{\mathbf{1 6 , 1 7}}$ thus biomarkers that were currently used also led to misunderstandings under certain conditions. Therefore, the emerging role of lncRNAs as potential diagnostic biomarkers maybe shine insight on the antidiastole on GCTs phenotypes.

In this study, two groups of patients (children and adult) which were stratified by two phenotypes of malignant GCTs (seminoma and yolk sac tumor) were involved. With the lncRNA classification pipeline, we firstly analyzed the differentially 
Table 2 Differentially expressed IncRNAs between children and adult

\begin{tabular}{|c|c|c|c|c|c|}
\hline Probesets & $\begin{array}{l}\text { RefSeq transcript } \\
\text { ID }\end{array}$ & Ensembl gene ID & Gene symbol & Regulation $^{a}$ & Gene title \\
\hline \multicolumn{6}{|l|}{ Seminoma } \\
\hline 214218_s_at & NR_001564 & ENSG00000274655 & XIST & Down & $\mathrm{X}$ (inactive)-specific transcript \\
\hline 205833_s_at & NR_024617 & ENSG00000152931 & PART1 & Down & Prostate androgen-regulated transcript 1 \\
\hline 205833_s_at & NR_024617 & ENSG00000273701 & PART1 & Down & Prostate androgen-regulated transcript 1 \\
\hline 207161_at & NR_022006 & ENSG00000122548 & KIAA0087 & Down & KIAA0087 \\
\hline 206819_at & NR_003714 & ENSG00000197210 & POM121L9P & Down & POM121 membrane glycoprotein-like 9 \\
\hline 210794_s_at & NR_002766 & ENSG00000214548 & MEG3 & Down & Maternally expressed 3 \\
\hline 212732_at & NR_002766 & ENSG00000214548 & MEG3 & Down & Maternally expressed 3 \\
\hline 216722_at & NR_001559 & ENSG00000230265 & VENTXP1 & Down & VENT homeobox (Xenopus laevis) 1 \\
\hline 210241_s_at & NR_015381 & ENSG00000182165 & TP53TG1 & Down & TP53 target 1 \\
\hline 220399_at & NR_024321 & ENSG00000225880 & NCRNA00115 & Down & Non-protein coding RNA 115 \\
\hline 214839_at & NR_024281 & ENSG00000253230 & LOC157627 & Down & Hypothetical LOC157627 \\
\hline 206819_at & NR_003714 & ENSG00000128262 & POM121L9P & Down & POM121 membrane glycoprotein-like 9 \\
\hline 216786_at & NR_002161 & ENSG00000230663 & NCRNA00230A & Down & Non-protein coding RNA $230 \mathrm{~A}$ \\
\hline 217506_at & NR_015389 & ENSG00000263753 & LOC339290 & Down & Hypothetical LOC339290 \\
\hline 216722_at & NR_001559 & ENSG00000259849 & VENTXP1 & Down & VENT homeobox (Xenopus laevis) 1 \\
\hline 216596_at & NR_026771 & ENSG00000225930 & DKFZP434L187 & Down & Hypothetical LOC26082 \\
\hline 220399_at & NR_024321 & ENSG00000272812 & NCRNA00115 & Down & Non-protein coding RNA 115 \\
\hline 214983_at & NR_001545 & ENSG00000233864 & TTTY15 & Up & Testis-specific transcript, Y-linked 15 \\
\hline 216596_at & NR_026771 & ENSG00000282096 & DKFZP434L187 & Down & Hypothetical LOC26082 \\
\hline
\end{tabular}

Yolk sac

206478_at

215972_at

222001_x_at

221129_at

222021_x_at

222021_x_at

222021_x_at

222021_x_at

222021_x_at

216786_at

206819_at

210794_s_at

212732_at

222021_x_at

222001_x_at

222021_x_at

210241_s_at

206819_at

222021_x_at

222021_x_at

222021_x_at

216053_x_at

222021_x_at

216053_x_at

214839_at

206819_at

216786_at

206478_at

222021_x_at

222021_x_at

215283 at

222021_x_at

222021_x_at
NR_026800
NR_024617
NR_024510
NR_026770
NR_003264
NR_003264
NR_003264
NR_003264
NR_003264
NR_002161
NR 003714
NR_002766
NR_002766
NR_003264
NR_024510
NR_003264
NR_015381
NR_003714
NR_003264
NR_003264
NR_003264
NR_026713
NR_003264
NR_026713
NR_024281
NR_003714
NR_002161
NR_026800
NR_003264
NR_003264
NR_015389
NR_003264
NR_003264

ENSG00000226777

ENSG00000152931

ENSG00000277147

ENSG00000267496

ENSG00000281237

ENSG00000242086

ENSG00000281794

ENSG00000215837

ENSG00000280912

ENSG00000233522

ENSG00000197210

ENSG00000214548

ENSG00000214548

ENSG00000280512

ENSG00000226067

ENSG00000281687

ENSG00000182165

ENSG00000161103

ENSG00000281334

ENSG00000073578

ENSG00000282953

ENSG00000125804

ENSG00000281915

ENSG00000175170

ENSG00000253230

ENSG00000128262

ENSG00000230663

ENSG00000277059

ENSG00000280521

ENSG00000280993

ENSG00000263753

ENSG00000280909

ENSG00000281060

\begin{tabular}{ll} 
KIAA0125 & Up \\
PART1 & Down \\
LOC728855 & Up \\
C17orf88 & Down \\
SDHAP1 & Down \\
SDHAP1 & Down \\
SDHAP1 & Down \\
SDHAP1 & Down \\
SDHAP1 & Down \\
NCRNA00230A & Down \\
POM121L9P & Down \\
MEG3 & Down \\
MEG3 & Down \\
SDHAP1 & Down \\
LOC728855 & Up \\
SDHAP1 & Down \\
TP53TG1 & Down \\
POM121L9P & Down \\
SDHAP1 & Down \\
SDHAP1 & Down \\
SDHAP1 & Down \\
FAM182A & Down \\
SDHAP1 & Down \\
FAM182A & Down \\
LOC157627 & Down \\
POM121L9P & Down \\
NCRNA00230A & Down \\
KIAA0125 & Up \\
SDHAP1 & Down \\
SDHAP1 & Down \\
LOC339290 & Up \\
SDHAP1 & Down \\
SDHAP1 & Down \\
\hline
\end{tabular}

\section{KIAA0125}

Prostate androgen-regulated transcript 1 Hypothetical LOC728855

Chromosome 17 open reading frame 88 Succinate dehydrogenase complex Succinate dehydrogenase complex Succinate dehydrogenase complex Succinate dehydrogenase complex Succinate dehydrogenase complex Non-protein coding RNA 230A POM121 membrane glycoprotein-like 9 Maternally expressed 3

Maternally expressed 3

Succinate dehydrogenase complex Hypothetical LOC728855

Succinate dehydrogenase complex TP53 target 1

POM121 membrane glycoprotein-like 9 Succinate dehydrogenase complex Succinate dehydrogenase complex Succinate dehydrogenase complex Family with sequence similarity 182 Succinate dehydrogenase complex Family with sequence similarity 182 Hypothetical LOC157627

POM121 membrane glycoprotein-like 9 Non-protein coding RNA 230A KIAA0125

Succinate dehydrogenase complex Succinate dehydrogenase complex Hypothetical LOC339290

Succinate dehydrogenase complex Succinate dehydrogenase complex 
Table 2 (Contd.)

\begin{tabular}{|c|c|c|c|c|c|}
\hline Probesets & $\begin{array}{l}\text { RefSeq transcript } \\
\text { ID }\end{array}$ & Ensembl gene ID & Gene symbol & Regulation $^{a}$ & Gene title \\
\hline 222021_x_at & NR_003264 & ENSG00000281603 & SDHAP1 & Down & Succinate dehydrogenase complex \\
\hline 216596_at & NR_026771 & ENSG00000225930 & DKFZP434L187 & Down & Hypothetical LOC26082 \\
\hline 64432_at & NR_015404 & ENSG00000234608 & C12orf47 & Down & Chromosome 12 open reading frame 47 \\
\hline 220324_at & NR_026807 & ENSG00000233237 & C6orf155 & Down & Chromosome 6 open reading frame 155 \\
\hline 216596_at & NR_026771 & ENSG00000282096 & DKFZP434L187 & Down & Hypothetical LOC26082 \\
\hline
\end{tabular}

expressed lncRNAs between seminoma and yolk sac tumor. A set of 11 aberrantly expressed lncRNAs were identified in adult and 12 lncRNAs were determined in children. Five distinctive lncRNAs including XIST, C17orf86, FAM182A, FLJ11235, and C12orf47 were involved in the intersection of children and adult, indicating a potential role of these lncRNAs in distinguishing seminoma from yolk sac tumor regardless of age. As reported previously, XIST expressions were widely detected in seminomatous testicular germ cell tumors, and the presence of the unmethylated XIST were frequent in testicular germ cell tumors. ${ }^{18}$ To our best knowledge, the role of the other four lncRNAs in malignant GCTs has not been investigated. However, the IncRNA C17orf86 that was also known as SNHG20 was associated with the metastasis of hepatocellular carcinoma, and the elevated expression level of SNHG20 could promote carcinoma cellular invasion. ${ }^{\mathbf{1 9 , 2 0}}$ Besides, the function of the left three IncRNAs including FAM182A, FLJ11235, and C12orf47 has not been explored even in other diseases.

Table 3 Differentially expressed IncRNAs between seminoma and yolk sac tumor despite of age

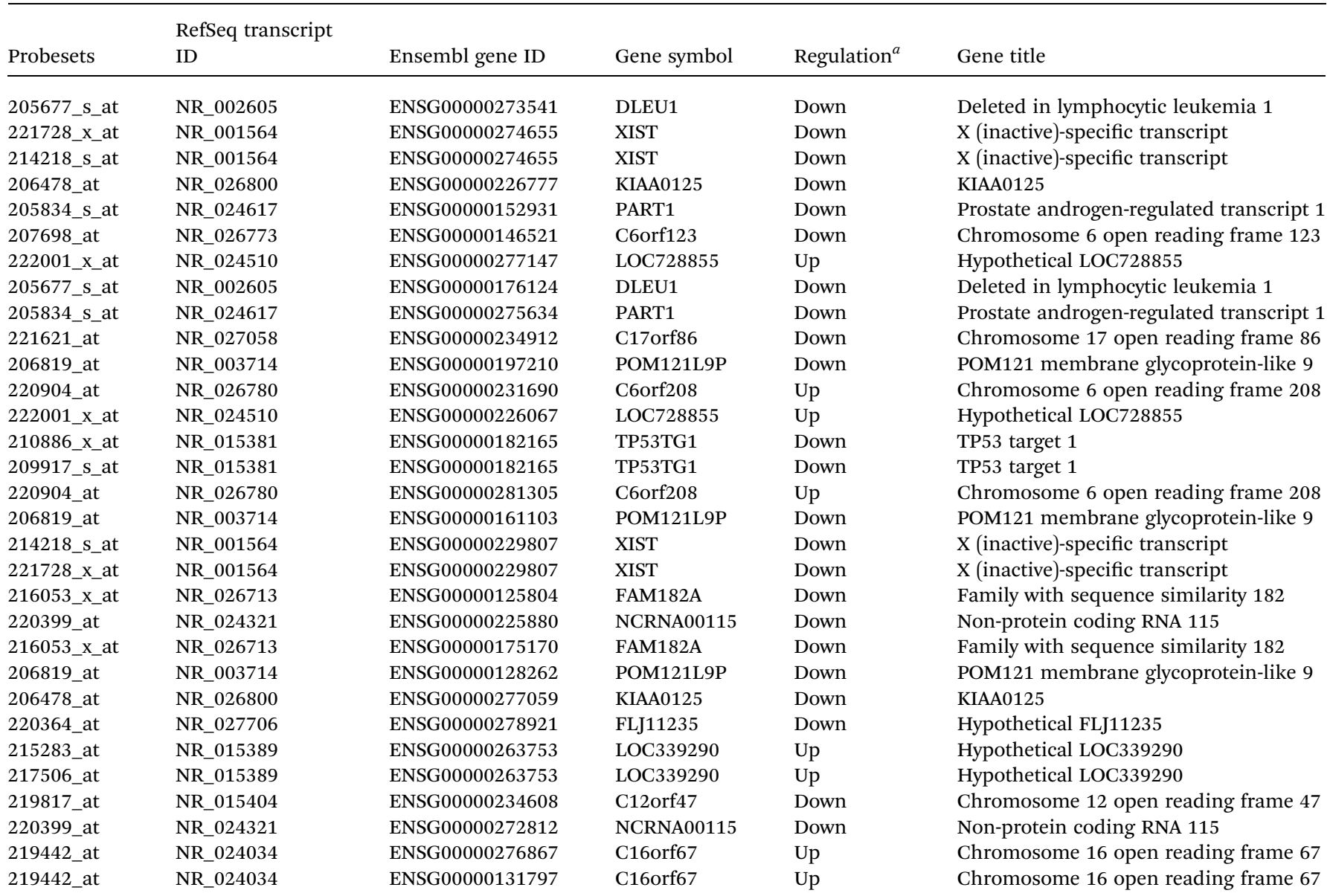

${ }^{a}$ Compared with seminoma. 
A

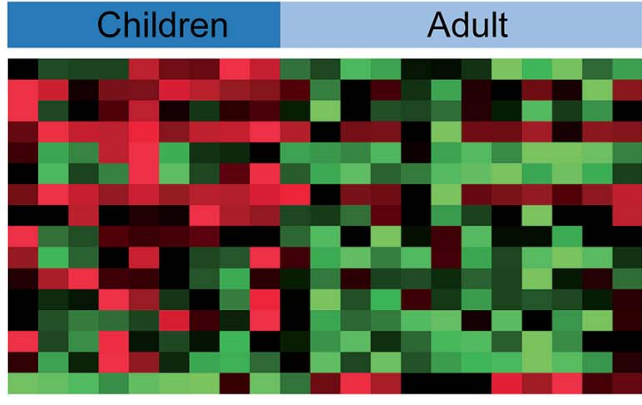

B

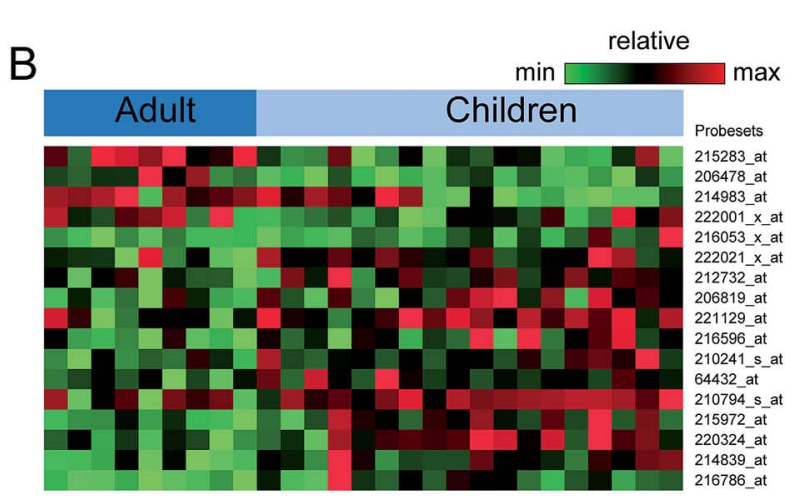

Probesets

217506_at

210794_s_at

221728_x_at

206819_at

205833_s_at

214218_s_at

210241_s_at

207259_at

214839_at

220399_at

216786_at

212732_at

207161_a

216722_at

14983_a

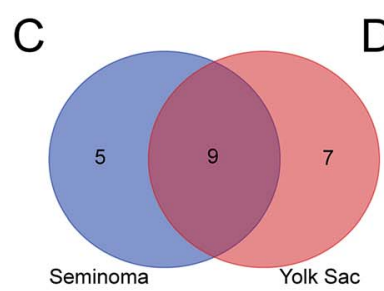

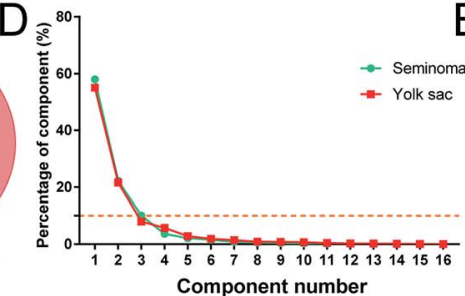

Component number

Fig. 5 Distinctive IncRNAs between adult and children. The distinctive IncRNAs between adult and children stratified by different clinical phenotypes of malignant germ cell tumors were identified ((A) seminoma, (B) yolk sac tumor), the IncRNAs that were relatively high expressed were shown in red, the lower expressed ones were shown in green. The overlapped IncRNAs between seminoma and yolk sac tumor were described with the shadow region in the Venn diagram (C). The results of principal component analysis were shown in (D-F). The percentage of every component was shown in (D), the lower control limit (10\%) for the principal component analysis were dotted in yellow, and two classes of the samples corresponding to the dots of different colors in 3D images were clustered ((E) seminoma, (F) yolk sac tumor, adult: green, children: yellow).

We also processed comparisons between children and adult, which were stratified by two phenotypes of GCTs (seminoma and yolk sac tumor). In total, 14 expressed lncRNAs were identified in seminoma and 16 lncRNAs were determined in yolk sac tumor. Of these, 9 lncRNAs including PART1, NCRNA00230A, POM121L9P, MEG3, TP53TG1, LOC157627, LOC339290, DKFZP434L187, and TTTY15 were overlapped between seminoma and yolk sac tumor, which suggested that people with one or a certain number of the overlapped lncRNAs may be facing high risk in suffering from seminoma or yolk sac tumor. Coincident with previous studies, MEG3, which has been widely found in many cancers, also regulated the growth of testicular germ cell tumor through PTEN/PI3K/AKT pathway. ${ }^{21}$ And the IncRNA POM121L9P was pointed out to be associated with male sterility via binding to Piwi proteins in mammalian. ${ }^{22}$ Another IncRNA, PART1, is a novel human prostate-specific and androgen-regulated gene that loci in chromosome 5q12, the available studies have proved that the expression level of this lncRNA was elevated by approximate $73.1 \%$ detected using specimens of stage I-III non-small cell lung cancer. ${ }^{23}$ Moreover,
TP53TG1 was an important regulator of cellular homeostasis, which could undergo cancer-specific promoter hypermethylation-associated silencing and inhibit the occurrence and development of cancer. ${ }^{24}$ Another lncRNA, TTTY15, was highly cited in prostate cancer, the fusion action mode of this gene with USP9Y was identified in a large cohort study of prostate cancer. $^{25-27}$ The function of the left lncRNAs is still lack of annotations.

To further explore identical biomarkers that were unaffected by age and GCTs phenotypes, we collaborated lncRNAs shared by groups of different ages or GCTs phenotypes, but none lncRNA fed back when we loaded these two datasets into the statistical software to compare for the overlapped region. However, in the validation process conducted by investigating Oncomine database, we found IncRNA XIST was differentially expressed between cancer and normal, indicating a IncRNA screening criteria of high strict and accurate hold by this study.

In summary, five differentially expressed lncRNAs shared by adult and child were identified in comparison between seminoma and yolk sac tumor, while nine lncRNAs shared by

Table 4 Comparison of IncRNA expressions between cancer and normal

\begin{tabular}{|c|c|c|c|c|c|c|}
\hline \multirow[b]{2}{*}{ lncRNA } & \multicolumn{3}{|l|}{ Seminoma } & \multicolumn{3}{|c|}{ Yolk sac tumor } \\
\hline & Fold change & $t$ value & $p$ value & Fold change & $t$ value & $p$ value \\
\hline C17orf86 & 1.23 & 2.90 & 0.01 & 1.00 & 0.02 & 0.49 \\
\hline
\end{tabular}


seminoma and yolk sac tumor were determined in comparison between adult and child. The IncRNAs identified in this study may be of great potential in distinguishing GCTs of different phenotypes (seminoma and yolk sac tumor), and they can also be used as promising biomarkers in indicating risk levels from which patients of seminoma or yolk sac tumor may suffer regardless of age. Although some of the lncRNAs had been validated, the majority of them have not been investigated, further studies are still needed.

\section{Conflicts of interest}

There are no conflicts of interests.

\section{Acknowledgements}

This research was supported by the National Natural Science Foundation of China (No. 81273039, 81472954 and 81773404). We thank those who helped us a lot in School of Public Health, Zhengzhou University.

\section{References}

1 R. L. Siegel, K. D. Miller and A. Jemal, Cancer statistics, 2016, Ca-Cancer J. Clin., 2016, 66, 7-30.

2 W. Chen, R. Zheng, P. D. Baade, et al., Cancer statistics in China, 2015, Ca-Cancer J. Clin., 2016, 66, 115-132.

3 K. D. Miller, R. L. Siegel, C. C. Lin, et al., Cancer treatment and survivorship statistics, 2016, Ca-Cancer J. Clin., 2016, 66, 271-289.

4 S. Zhang, G. Liang, Y. Ju and C. You, Clinical and Radiologic Features of Pediatric Basal Ganglia Germ Cell Tumors, World Neurosurg., 2016, 95, 516-524.

5 U. Gobel, D. T. Schneider, G. Calaminus, R. J. Haas, P. Schmidt and D. Harms, Germ-cell tumors in childhood and adolescence. GPOH MAKEI and the MAHO study groups, Ann. Oncol., 2000, 11, 263-271.

6 F. J. Rescorla, Pediatric germ cell tumors, Semin. Surg. Oncol., 1999, 16, 144.

7 G. Teilum, Classification of endodermal sinus tumour (mesoblastoma vitellinum) and so-called "embryonal carcinoma" of the ovary, Acta Pathol. Microbiol. Scand., 1965, 64, 407.

8 I. Juric and N. Basic-Jukic, Testicular Seminoma Occurring After Kidney Transplantation in a Patient Previously Treated for Teratoma: De Novo Malignancy or Recurrence in a Different Histologic Form?, Transplant. Proc., 2016, 48, 3128-3129.

9 H. Zhang, P. Zhang, J. Fan, et al., Determining an Optimal Cutoff of Serum beta-Human Chorionic Gonadotropin for Assisting the Diagnosis of Intracranial Germinomas, PLoS One, 2016, 11, e0147023.

10 Y. Huang, Z. Jia, J. Tu, T. Shen, F. Tian and G. Jiang, Supplemental conventional transarterial embolization/ chemoembolization therapy via extrahepatic arteries for hepatocellular carcinoma, J. Cancer Res. Ther., 2017, 13, 720-724.
$11 \mathrm{~J}$. J. Quinn and H. Y. Chang, Unique features of long noncoding RNA biogenesis and function, Nat. Rev. Genet, 2016, 17, 47-62.

12 X. Su, G. G. Malouf, Y. Chen, et al., Comprehensive analysis of long non-coding RNAs in human breast cancer clinical subtypes, Oncotarget, 2014, 5, 9864-9876.

13 F. Wu, C. Zhang, J. Cai, et al., Upregulation of long noncoding RNA HOXA-AS3 promotes tumor progression and predicts poor prognosis in glioma, Oncotarget, 2017, 8, 53110-53123.

14 J. Li, Z. Li, W. Zheng, et al., LncRNA-ATB: an indispensable cancer-related long noncoding RNA, Cell Proliferation, 2017, 50, e12381.

15 X. Zhang, S. Sun, J. K. Pu, et al., Long non-coding RNA expression profiles predict clinical phenotypes in glioma, Neurobiol. Dis., 2012, 48, 1-8.

16 J. M. Bieniek, T. Juvet, M. Margolis, E. D. Grober, K. C. Lo and K. A. Jarvi, Prevalence and management of incidental small testicular masses discovered on ultrasonographic evaluation of male infertility, J. Urol., in press.

17 W. Xu and Y. Li, Is Omentectomy Mandatory Among Early Stage (I, II) Malignant Ovarian Germ Cell Tumor Patients? A Retrospective Study of 223 Cases, Int. J. Gynecol. Cancer, 2017, 27, 1373-1378.

18 G. E. Lind, R. I. Skotheim and R. A. Lothe, The epigenome of testicular germ cell tumors, APMIS, 2007, 115, 1147-1160.

19 D. Zhang, C. Cao, L. Liu and D. Wu, Up-regulation of LncRNA SNHG20 Predicts Poor Prognosis in Hepatocellular Carcinoma, J. Cancer, 2016, 7, 608-617.

20 J. Liu, C. Lu, M. Xiao, F. Jiang, L. Qu and R. Ni, Long non-coding RNA SNHG20 predicts a poor prognosis for HCC and promotes cell invasion by regulating the epithelial-to-mesenchymal transition, Biomed. Pharmacother., 2017, 89, 857-863.

21 N. Q. Yang, X. J. Luo, J. Zhang, G. M. Wang and J. M. Guo, Crosstalk between Meg3 and miR-1297 regulates growth of testicular germ cell tumor through PTEN/PI3K/AKT pathway, Am. J. Transl. Res., 2016, 8, 1091-1099.

22 A. Girard, R. Sachidanandam, G. J. Hannon and M. A. Carmell, A germline-specific class of small RNAs binds mammalian Piwi proteins, Nature, 2006, 442, 199-202.

23 M. Li, W. Zhang, S. Zhang, C. Wang and Y. Lin, PART1 expression is associated with poor prognosis and tumor recurrence in stage I-III non-small cell lung cancer, $J$. Cancer, 2017, 8, 1795-1800.

24 A. Diaz-Lagares, A. B. Crujeiras, P. Lopez-Serra, et al., Epigenetic inactivation of the p53-induced long noncoding RNA TP53 target 1 in human cancer, Proc. Natl. Acad. Sci. U. S. A., 2016, 113, E7535-E7544.

25 Y. Zhang, X. Y. Mao, X. Liu, et al., High frequency of the SDK1:AMACR fusion transcript in Chinese prostate cancer, Int. J. Clin. Exp. Med., 2015, 8, 15127-15136.

26 Y. Zhu, S. Ren, T. Jing, et al., Clinical utility of a novel urinebased gene fusion TTTY15-USP9Y in predicting prostate biopsy outcome, Urol. Oncol., 2015, 33, 384.

27 S. Ren, Z. Peng, J. H. Mao, et al., RNA-seq analysis of prostate cancer in the Chinese population identifies recurrent gene fusions, cancer-associated long noncoding RNAs and aberrant alternative splicings, Cell Res., 2012, 22, 806-821. 